\title{
ORDERED STATES IN THE DISORDERED HUBBARD MODEL
}

\author{
P. J. H. Denteneer* \\ Instituut-Lorentz, University of Leiden, \\ P. O. Box 9506, 2300 RA Leiden, The Netherlands, \\ M. Ulmke \\ Theoretische Physik III, Universität Augsburg, \\ D 86135 Augsburg, Germany \\ R. T. Scalettar, G. T. Zimanyi \\ Department of Physics, University of California, \\ Davis, CA 95616, USA
}

\begin{abstract}
The Hubbard model is studied in which disorder is introduced by putting the on-site interaction to zero on a fraction $f$ of (impurity) sites of a square lattice. Using Quantum Monte Carlo methods and Dynamical Mean-Field Theory we find that antiferromagnetic long-range order is initially enhanced at half-filling and stabilized off half-filling by the disorder. The Mott-Hubbard charge gap of the pure system is broken up in two pieces by the disorder: one incompressible state remains at average density $n=1$ and another can be seen slightly below $n=1+f$. Qualitative explanations are provided.
\end{abstract}

PACS: 75.10.Lp, 71.23.-k, 71.27.+a, 75.40.Mg

Short title: Order in the disordered Hubbard model

*e-mail address: pjhdent@lorentz.leidenuniv.nl 


\section{Introduction}

The problem of the interplay between disorder and interactions in systems of electrons is challenging and has a long history [1]. Disorder can, by itself, localize electrons and make the conductivity disappear, the Anderson transition. At appropriate densities, interactions can also cause the formation of insulating states via the Mott transition. In the latter case, the insulating states often possess additional magnetic or charge ordering. The simultaneous presence of disorder and interactions is realized in many systems in nature, with fundamental and fascinating consequences, e.g. the unusual magnetic properties of heavily doped, compensated semiconductors, and the unique transport properties of two-dimensional and bulk superconductors [1]. If both disorder and interactions are present, the effects can sometimes reinforce each other, but can also compete. In classical spin models, it has recently become clear that quenched disorder can turn (temperature-driven) symmetry-breaking first order phase transitions into continuous phase transitions, possibly with new intervening critical points [2]. Although the arguments do not appear to apply directly to interacting fermions, disorder may lead to similarly interesting phenomena.

The Hubbard model for interacting electrons on a lattice [3] exhibits both Mott metal-insulator and magnetic phase transitions. On the one hand, it is expected that the interaction $U$ induces a gap at half-filling by separating many-body states with doubly occupied orbitals from those with holes, when the on-site repulsion becomes larger than the non-interacting bandwidth. On the other hand, also near half-filling, there is a tendency to antiferromagnetic (AF) ordering of the electron spins. One could now ask the question whether the Mott transition in fact ever occurs in the absence of some associated symmetry breaking such as magnetic order [四]. For interacting bosons the Mott transition (in this case from a superfluid) to an insulator is known to occur without any other accompanying order parameter [5].

To investigate the behavior of disordered interacting electron systems it is of interest to consider the Hubbard Hamiltonian into which disorder is introduced:

$$
\hat{H}_{\mathrm{H}}=-\sum_{i, j, \sigma} t_{i j} c_{i \sigma}^{\dagger} c_{j \sigma}+\sum_{j} U_{j}\left(n_{j \uparrow}-\frac{1}{2}\right)\left(n_{j \downarrow}-\frac{1}{2}\right)-\sum_{j, \sigma}\left(\mu-\varepsilon_{j}\right) n_{j \sigma},
$$

where $c_{j \sigma}$ is the annihilation operator for an electron at site $j$ with spin $\sigma$. $t_{i j}$ is the hopping integral (non-zero only between neighboring sites $i$ and $j$ ), $U_{j}$ the on-site interaction at site $j$ between electrons of opposite spin, and $n_{j \sigma}=c_{j \sigma}^{\dagger} c_{j \sigma}$ is the occupation number operator. Various kinds of disorder are allowed for in (11): (i) in the hopping integrals $t_{i j}$, (ii) in the on-site energy $\varepsilon_{j}$, or (iii) in the onsite interaction $U_{j}$. Traditionally, because the case of non-interacting electrons was considered first, attention has been devoted to the first two kinds of disorder, termed off-diagonal and diagonal disorder, respectively. In recent Quantum Monte Carlo simulations, the first two kinds were studied including uniform interactions, showing the destruction of AF long-range order at half-filling for some critical strength of disorder in case (i), and allowing for few conclusions in case (ii) because of sign problems already at half-filling [6]. In the present paper, we study case (iii), i.e. the 
Hamiltonian:

$$
\hat{H}=-t \sum_{\langle i, j\rangle, \sigma} c_{i \sigma}^{\dagger} c_{j \sigma}+\sum_{j} U_{j}\left(n_{j \uparrow}-\frac{1}{2}\right)\left(n_{j \downarrow}-\frac{1}{2}\right)-\mu \sum_{j, \sigma} n_{j \sigma},
$$

in which the on-site repulsion $U_{j}$ is turned off from $U$ on a fraction $f$ of all sites $(\langle i, j\rangle$ denotes neighboring sites $i$ and $j$ ), i.e. the $U_{j}$ are taken from a bimodal distribution,

$$
P\left(U_{j}\right)=(1-f) \delta\left(U_{j}-U\right)+f \delta\left(U_{j}\right) .
$$

A physical realization of this kind of disorder could be impurity atoms in a crystal which can accomodate two electrons with a strongly reduced Coulomb repulsion, e.g. non-magnetic impurities (like $\mathrm{Zn}$ ) replacing copper atoms in the copper oxides. Here, we do not want to attach ourselves to a specific physical system, but try to understand what happens to ordered states of interacting electrons when disorder of kind (iii) is introduced. One expects the Mott transition to be shifted to average density $n=1+f$ (at which density also the $U \neq 0$ sites start to be doubly occupied), so that it is perhaps separated from the AF magnetic order which is likely to stay at $n=1$. New types of ordered states may also result.

To explore these phenomena tractable and reliable theoretical methods are hard to come by. Specifically, mean-field approaches to the physics of strongly correlated quantum systems, like the Hartree-Fock approximation and the slave-boson mean-field approach, can provide significant insight into the possible phases which can arise due to interaction effects, but are well-known to overestimate significantly the tendency to form ordered states [7, 8]. Other analytical approaches like the renormalization group, while powerful, likewise have their limitations [1]. The most obvious numerical approach, exact diagonalization of the Hamiltonian, is restricted to small lattices (10-20 particles) [9]. In this paper, we intend to study the effects of interactions and randomness by means of a stochastic sampling of the state space, the Quantum Monte Carlo (QMC) method. In this approach, correlation effects are treated exactly. A few hundred interacting electrons can be simulated, roughly an order of magnitude larger than by exact diagonalization. While the constraints of this finite size are still very significant, this number of particles is often sufficient to allow systematic extrapolations to bulk behavior, at least in two dimensions. The QMC method we use is the Determinant Monte Carlo method [10], which has been extensively applied to non-random interacting fermion Hamiltonians. We combine these simulations with calculations using Dynamical Mean-Field Theory (DMFT), or: the infinite-dimension approach [11. This technique improves significantly upon the Hartree-Fock approximation since it is non-perturbative and takes quantum fluctuations into account via a dynamical self-energy. The infinitedimension approach allows us to analyze problems in the thermodynamic limit and in larger ranges of parameter space, e.g. lower temperatures, than accessible to the determinant algorithm. DMFT has provided valuable insight into the physics of strongly correlated electron systems, e.g. the Mott-Hubbard transition [4, 11]. Both techniques, which recently have been applied to disordered Hubbard models also [6, 12, 13, will be briefly described in Section 2. In subsequent sections, we introduce the quantities of interest that are calculated, present results and discuss them. 


\section{Quantum Monte Carlo and Dynamical Mean Field Theory}

\subsection{Determinant Monte Carlo method}

A brief description of the Determinant Monte Carlo algorithm for the Hubbard Hamiltonian (2) is given. One is interested in computing operator expectation values like:

$$
\langle\hat{A}\rangle=\frac{\operatorname{Tr} \hat{A} e^{-\beta \hat{H}}}{\operatorname{Tr} e^{-\beta \hat{H}}} .
$$

The trace over the fermion degrees of freedom cannot be performed analytically due to the quartic interaction term. In order to reduce the problem to a quadratic Hamiltonian we discretize, $\beta=L_{\tau} \Delta \tau$, and employ the Trotter decomposition [14]. The partition function can now be expressed as:

$$
Z=\operatorname{Tr} e^{-\beta \hat{H}}=\operatorname{Tr}\left[e^{-\Delta \tau \hat{H}}\right]^{L_{\tau}} \approx \operatorname{Tr}\left[e^{-\Delta \tau \hat{K}} e^{-\Delta \tau \hat{P}}\right]^{L_{\tau}} .
$$

Here $\hat{K}$ includes the a priori quadratic pieces of $\hat{H}$, the hopping and chemical potential terms, while $\hat{P}$ is the on-site interaction term. In the second step, errors in measured quantities of order $t U(\Delta \tau)^{2}$ are introduced. Now that $\hat{P}$ has been isolated, the discrete Hubbard-Stratonovich transformation of Hirsch [15] can be used to decouple the interaction. If $U>0$,

$$
e^{-\Delta \tau U\left(n_{i \uparrow}-\frac{1}{2}\right)\left(n_{i \downarrow}-\frac{1}{2}\right)}=\frac{1}{2} e^{-U \Delta \tau / 4} \sum_{S_{i, \tau}= \pm 1} e^{-\lambda \Delta \tau S_{i, \tau}\left(n_{i \uparrow}-n_{i \downarrow}\right)} .
$$

Here $\cosh (\lambda \Delta \tau)=\exp (U \Delta \tau / 2)$. A field variable $S_{i, \tau}$ (of Ising type) must be introduced at each lattice site and imaginary time slice. If $U<0$ the field $S_{i, \tau}$ couples to the charge $n_{i \uparrow}+n_{i \downarrow}$ on each site. In either case, the end result is that the partition function can be rewritten as:

$$
Z=\sum_{S_{i, \tau}} \operatorname{Tr} \prod_{l} e^{-\hat{K}} e^{-\hat{P}^{\prime}(l)},
$$

where the fermion operators appear only quadratically in the transformed $\hat{P}^{\prime}$ and $l$ denotes the Trotter time slice. The interacting fermions have been replaced by non-interacting fermions evolving in a space- and time-dependent field. Performing the fermion trace is now possible, and results in:

$$
Z=\sum_{S_{i, \tau}} \operatorname{det} M_{\uparrow}\left(S_{i, \tau}\right) \operatorname{det} M_{\downarrow}\left(S_{i, \tau}\right),
$$

where the detailed forms of the matrices $M_{\sigma}$, which have dimension the number of spatial lattice sites $N$, are written down by Blankenbecler et al. [10].

One crucial bottleneck in the algorithm is that while at high temperatures the product of the fermion determinants is positive and can be used as a statistical weight, at low temperatures (high $\beta$ ) when $U>0$ and the system is away from half-filling, this is no longer always true. In this case, as $\beta$ increases the product 
can become negative. This is referred to as the "sign problem". The sign problem precludes simulations for large lattices at low temperatures. When $U<0$ no sign problem occurs, and this is one reason the attractive Hubbard model is easier to study.

The Monte Carlo simulation proceeds by going through the space-time lattice of $S_{i, \tau}$ and computing the ratio of the product of fermion determinants before and after a single spin is flipped. Naively, the simulation time scales as the fourth power of the number of sites $N$. One power of $N$ comes from updating all the sites. For each such update, the calculation of the determinant is of order $N^{3}$. However, one can reformulate the algorithm to scale with one lower power of $N$ as follows: instead of evaluating the new determinant from scratch, the ratio of the determinants may be evaluated in terms of the elements of $M_{\sigma}^{-1}$, the fermion Green function. One then must keep track of $M_{\sigma}^{-1}$, which of course changes as moves are accepted. However, the update of $M_{\sigma}^{-1}$ resulting from a change to $M_{\sigma}$ is only an $N^{2}$ procedure, due to the specific local nature of the change in $M_{\sigma}$. In summary, the technique involves the manipulation (mostly multiplication) of matrices of dimension the number of spatial sites in the system. This number may be up to several hundred on presentday computers.

The inputs to the computer code are the quantities that specify the physical system. They are: the spatial size of the system and the inverse temperature $\beta$. Also, the parameters in Hamiltonian (2) $, t, U_{j}$, and $\mu$, need to be specified. Finally, the parameters of the simulation, the Trotter time interval $\Delta \tau$ (which then determines the number of time steps $L_{\tau}$ ), the number of warm-up and measurement sweeps, the time between measurements etc. must be selected. Quite some care needs to be taken in determining these latter quantities to ensure the simulation results are meaningful.

One crucial feature of the Determinant Monte Carlo simulations is that the fermion Green functions $G_{i j}\left(\tau, \tau^{\prime}\right)=\left\langle T c_{i}(\tau) c_{j}^{\dagger}\left(\tau^{\prime}\right)\right\rangle$ are readily obtainable. In fact, $G$ is just the inverse of the matrix $M$ whose determinant gives the Boltzmann weight. It is available "free of charge" (at least for $\tau=\tau^{\prime}$ ) in the sense that it has already been constructed in moving the field variables. With the single-particle Green function in hand, we can measure quantities involving more than two fermion operators by performing the appropriate Wick contractions and expressing them in terms of products of single-particle propagators. There is considerable flexibility in the quantities one can calculate, and it is possible to decompose diagrams and separate self-energy and vertex contributions. This is useful when comparing quantum simulations with analytic calculations [16].

The Determinant Monte Carlo algorithm as described above has been extensively used for over a decade now. The more reliable applications have mainly been due to a number of recent technical advances. Here we only mention the most important one for our present studies: the introduction of matrix stabilization procedures has significantly improved the ability to reach low temperatures [17, 18]. With these techniques inverse temperatures as high as $\beta t=20$ can be attained.

In the present study, we have used $U=8 t$ (equal to the band width) combined with a Trotter time step $\Delta \tau=1 / 12 t$, ensuring small Trotter errors. Experience from previous studies [18] and independent tests in the present work teach that 
an inverse temperature $\beta t=8$ is sufficiently high to effectively yield ground-state thermodynamic and correlation functions on the lattices considered. This temperature is used throughout. We typically use 200 to 300 warm-up sweeps and 2000 to 4000 measurement sweeps. A simplifying detail of the present problem, in which a fraction $f$ of the sites have $U=0$, is that the corresponding Hubbard-Stratonovich coupling $\lambda$ in (6) equals zero and the local electron Green function does not need updating. We study square lattices with linear size up to $L=10$ and average over up to 40 disorder realizations.

Like the original Hubbard model, Hamiltonian (2) preserves a particle-hole symmetry at half-filling $(n=1$ and $\mu=0)$, i.e. it is invariant under the "staggered"

particle-hole transformation $c_{i \sigma}^{\dagger} \rightarrow(-1)^{i} c_{i \sigma}$. This symmetry ensures that there is no sign problem at $n=1$. Note that the particle-hole symmetry corresponds to different chemical potentials on the two constituents. When going off half-filling we do encounter the sign problem. However, we find it to be less detrimental than in the case without disorder. Aided furthermore by the disorder averaging it is possible to obtain meaningful data off half-filling (see also section đ). We further support our findings by comparison to results of DMFT calculations.

\subsection{Dynamical Mean-Field Theory}

For classical spin models (e.g. the Ising model) it is well known that the Weiss molecular field (or: mean field) theory becomes exact in the limit of high spatial dimensions. For lattice electrons this limit was introduced only recently; with the proper scaling of the hopping element in (2),$t=t^{*} / \sqrt{z}$ ( $z$ is the number of nearest neighbors) it leads to a quantum mechanical dynamical mean-field theory (DMFT). Here we give a brief description of this approach to our problem at hand; more details on the method are found in the literature [11, 13].

Thermodynamic properties are determined from the averaged grand potential $\Omega_{\mathrm{av}}$ :

$$
\beta \Omega_{\mathrm{av}}=-\langle(\ln \operatorname{Tr} \exp (-\beta \hat{H}))\rangle_{\mathrm{av}},
$$

where $\langle\ldots\rangle_{\text {av }}$ denotes the disorder average. The main simplification in the limit $d \rightarrow \infty$ lies in the reduction of the average, which has to be performed only on a single site [19] (for each allowed value of $U_{j}$, and using a coherent potential type of approximation). The explicit expression for the grand potential in the paramagnetic phase is given by:

$$
\begin{aligned}
\beta \Omega_{\mathrm{av}} & =-N \sum_{\sigma n} \int_{-\infty}^{\infty} \mathrm{d} E N^{0}(E) \ln \left[i \omega_{n}+\mu-\Sigma_{\sigma n}-E\right] \\
& +N \sum_{\sigma n} \ln G_{\sigma n}^{-1}-N\left\langle\ln \mathcal{Z}\left\{G, \Sigma, U_{j}\right\}\right\rangle_{\mathrm{av}}
\end{aligned}
$$

where $\omega_{n}=(2 n+1) \pi / \beta$ are Matsubara frequencies and $N^{0}(E)$ is the density of states (DOS) of non-interacting electrons (scaled to be suitable for comparison with the square lattice [20]). In (10) also appear: the generalized atomic partition function $\mathcal{Z}$ (shown to be equivalent to that of a single-impurity Anderson model [22]), and the complex quantities $G_{\sigma n}$ and $\Sigma_{\sigma n}$ which at this point enter as variational parameters. 
The physical values of $G_{\sigma n}$ and $\Sigma_{\sigma n}$ (namely, the local Green function, $G_{\sigma n} \equiv G_{i i, \sigma n}$, and the electron self-energy, $\Sigma_{\sigma n} \equiv \Sigma_{i i, \sigma n}$, respectively) correspond to those for which (10) is stationary [21]. The stationarity conditions: $\delta \Omega_{a v} / \delta G_{\sigma n}=0, \delta \Omega_{a v} / \delta \Sigma_{\sigma n}=$ 0 , yield two coupled sets of self-consistent equations for $G_{\sigma n}$ and $\Sigma_{\sigma n}$ :

$$
\begin{aligned}
G_{\sigma n} & =-\int_{-\infty}^{\infty} \mathrm{d} E \frac{N^{0}(E)}{i \omega_{n}+\mu-\Sigma_{\sigma n}-E} \\
G_{\sigma n} & =\int_{0}^{\beta} \mathrm{d} \tau e^{i \omega_{n} \tau}\left\langle\left\langle c_{\sigma}(\tau) c_{\sigma}^{*}(0)\right\rangle_{T}\right\rangle_{\mathrm{av}}
\end{aligned}
$$

where $\langle\ldots\rangle_{T}$ denotes the thermal average (which depends on $G_{\sigma n}$ and $\Sigma_{\sigma n}$ implicitly). Physically, the Dyson equation (11) describes the local Green function of independent electrons moving in a homogeneous dynamical potential $\Sigma_{\sigma n}$. It can be solved by a simple integration for each Matsubara frequency. The functional integral (12) on the other hand is highly non-trivial since it couples all Matsubara frequencies. The interacting, disordered problem is mapped onto an ensemble of single-impurity problems, complemented by a self-consistency condition which introduces the lattice into the problem [12, [19]. We emphasize that, in contrast to conventional mean-field theories, in this dynamical mean-field theory (DMFT), the action remains time dependent, i.e. local fluctuations are retained.

The local interacting problem (12) is solved numerically using a finite-temperature, auxiliary-field Quantum Monte Carlo (QMC) method [23]. Quite similar as in the finite-dimensional QMC algorithm described above, the electron-electron interaction is formally replaced by an interaction of independent electrons with a dynamical, auxiliary field of Ising-type spins. To this end the interval $[0, \beta]$ is again discretized into $L_{\tau}$ steps of size $\Delta \tau=\beta / L_{\tau}$. Equivalently, there is a high energy cut-off of Matsubara frequencies, i.e. $\left|\omega_{n}\right|=|2 n+1| \pi / \beta<\pi / \Delta \tau, n=-L_{\tau} / 2, \ldots, L_{\tau} / 2-1$. The computer time grows like $L_{\tau}^{3} \propto \beta^{3}$, restricting $L_{\tau}$ to values below $\sim 150$ and $\beta t^{*} \leq 50 \ldots 70$ on present supercomputers. For small $L_{\tau}\left(L_{\tau} \leq 20\right)$ one can perform a full enumeration (instead of Monte Carlo sampling) of all $2^{L_{\tau}}$ possible configurations of the auxiliary field. Sign problems turn out to be absent in the DMFT approach.

Self-consistency is obtained iteratively as follows: the Green function $G$ (omitting indices) is calculated from some initial self energy, e.g. $\Sigma \equiv 0$, by the Dyson equation (11). Then, the new Green function $G_{\text {new }}$ is determined by solving (12) for each value of $U_{j}$ with the QMC method, and averaging. Finally, the calculation of the new self energy $\Sigma_{\text {new }}=\Sigma-G_{\text {new }}^{-1}+G^{-1}$ completes one iteration. Typically 10 iterations with $20000 \mathrm{MC}$ sweeps are necessary to obtain a convergence of $\sim 10^{-3}$. Close to a phase transition the convergence is much slower and the statistical errors are larger due to strong fluctuations. At large $U$-values $(U>12 t)$ the Monte Carlo sampling becomes more and more inefficient due to "sticking" problems, i.e. there are two (or more) minima in the free energy and the single spin-flip algorithm is no longer able to transfer between them.

The self-consistent equations (11) and (12) can easily be extended to a phase with AF long range order (For details of the implementation see [13]). One uses the 
property that, in the case of finite sublattice magnetization but zero total magnetization, i.e. excluding ferrimagnetism, the Green function for an $\uparrow-$ spin on sublattice $A$ is equal to that of a $\downarrow-$ spin on sublattice $B$ and vice versa.

\section{Quantities of Interest}

In this paper, we only study thermodynamic quantities and static correlation functions. At a later stage also (imaginary-) time-dependent correlation functions will be of interest, e.g. to study frequency-dependent response functions.

In the QMC calculations, the (equal-time) electron Green function $G_{i j \sigma}=$ $\left\langle c_{i \sigma} c_{j \sigma}^{\dagger}\right\rangle=M_{i j \sigma}^{-1}$, described in section 2.1 immediately leads to electron densities, $\left\langle n_{i \sigma}\right\rangle=1-G_{i i \sigma}$, and static correlation functions. The magnetic correlations which give crucial information on the physics of the Hubbard model can be monitored by measuring the equal-time spin-spin correlation function $c(l)=\left\langle\left(n_{j \uparrow}-n_{j \downarrow}\right)\left(n_{j+l \uparrow}-\right.\right.$ $\left.\left.n_{j+l \downarrow}\right)\right\rangle$ and its Fourier transform $S(q)=\sum_{l} e^{i q \cdot l} c(l)$, the magnetic structure factor. Specifically, a finite-size scaling study of $S(\pi, \pi)$ leads to the sublattice magnetization $M$ of the phase with AF long range order, according to spin wave theory [24]:

$$
\frac{S(\pi, \pi)}{L^{2}}=\frac{M^{2}}{3}+\mathcal{O}(1 / L)
$$

where $L$ is the linear system size.

In the DMFT calculations (see section 2.2), the resulting Green functions also lead to, for instance, the densities $n_{\sigma}$ and sublattice magnetization $M$. The Green function $G_{i i \sigma}$ is just the Fourier transform of $G_{\sigma n}$ in section 2.2. Note that in this approach quantities like the order parameter $M$ are calculated in the thermodynamic limit, so no finite-size scaling is required. The vanishing of $M$ is used to determine phase boundaries as a function of parameters like $\mu, U, T$, or $f$, leading to e.g. the Néel temperature $T_{\mathrm{N}}$. A different way to obtain these phase boundaries is to calculate the corresponding susceptibility $\chi_{\mathrm{AF}}$ (via two-particle correlation functions; see [13] for details). Divergence of $\chi_{\mathrm{AF}}$ signals a continuous phase transition.

\section{Results and Discussion}

\subsection{Magnetic order}

First, we study the effect of an increasing concentration $f$ of $U=0$ sites on the stability of AF long range order at $n=1$. The static AF structure factor $S(\pi, \pi)$ is calculated at $U=8 t$ for different lattice sizes and temperatures. For $L \leq 10$, $S(\pi, \pi)$ is found to saturate at $T \approx t / 8$. ¿From the saturated values the ground-state sublattice magnetization $M$ can be extrapolated using finite-size scaling, according to (13). Scaling plots for different $f$ at $U=8 t$ are shown in Fig. 1. For $f \leq 0.36$, $S(\pi, \pi) / L^{2}$ extrapolates to a finite value in the thermodynamic limit. Long range order has disappeared for $f \geq 0.5$. Fig. 2 presents the extrapolated values $M(f)$. For small disorder $M$ is found to increase with $f$, it reaches a maximum around $f=0.1$, and eventually vanishes at $f_{c} \approx 0.4$. This initial increase of the order parameter 


$$
T=t / 8 \quad U=8 t
$$

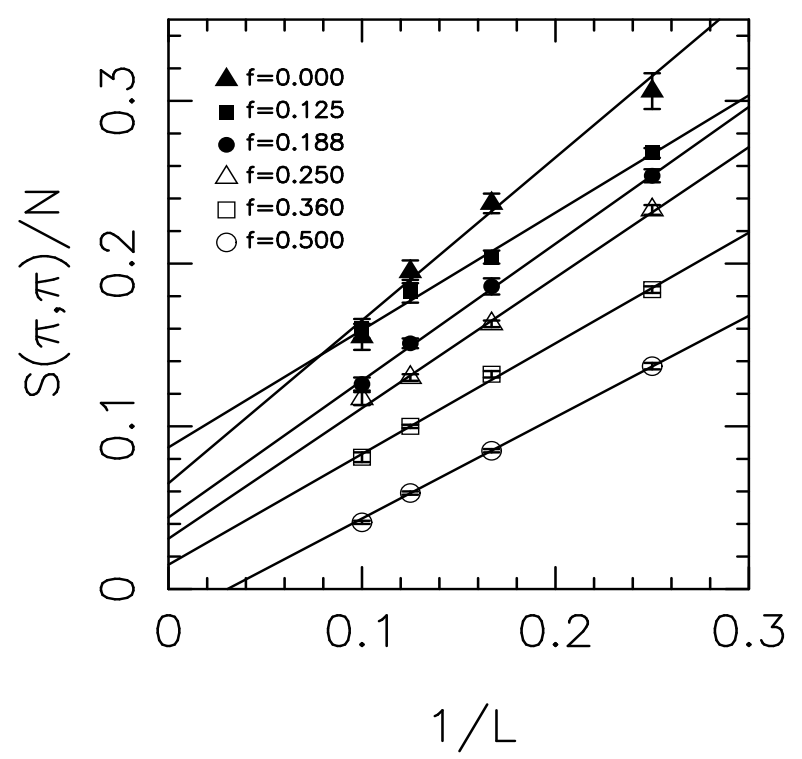

Figure 1: Finite-size scaling of the antiferromagnetic structure factor $S(\pi, \pi)$ on the square lattice at half-filling for $f=0,0.125,0.188,0.25,0.36,0.5$ (top to bottom; $f$ is the fraction of $U=0$ sites), computed using the Determinant QMC method with $U=8 t$ and $T=t / 8$. $L$ denotes the linear system size and $N$ the number of sites $\left(N=L^{2}\right)$. For values of $f$ that do not correspond to an integer number of defects, we have interpolated between the results for the two bracketing concentrations.

prior to the formation of a disordered phase is remarkable. Similar phenomena have however been seen in studies of the Hubbard model with random site energies in infinite dimensions (an initial increase with disorder of the critical temperature for $\mathrm{AF}$ order [13]). Local enhancement of AF fluctuations by vacancies has also been reported recently [25].

For the present model, at half-filling, we do not find initially enhanced AF order in the infinite-dimension approach as can be seen in Fig. 3 (Due to the neglect of spatial fluctuations, we do of course find that AF order survives longer when increasing disorder: $f_{c} \approx 0.75$. This can be higher than the percolation threshold because no sites are actually removed). On the other hand, also in this approach we can see that $\mathrm{AF}$ order is enhanced by introducing $U=0$ sites: we find that $\mathrm{AF}$ order occurs at densities for which the pure system is not ordered. A calculated $(f, n)$ phase diagram shows that AF order persists up to increasing values of $n$ (relative to $f=0$, for which in DMFT AF order occurs up to $n=1.14$, i.e. somewhat off halffilling) when the disorder increases from $f=0$ to about $f=0.36$ [26]. Therefore, again the presence of impurity sites stabilizes the magnetic order. We note that the calculation of this phase diagram using the Determinant QMC method in finite dimension is very hard, if not impossible, because of the sign problem. We, however, expect the phase boundary between AF and disordered phase to be of similar form (but shifted towards half-filling). 


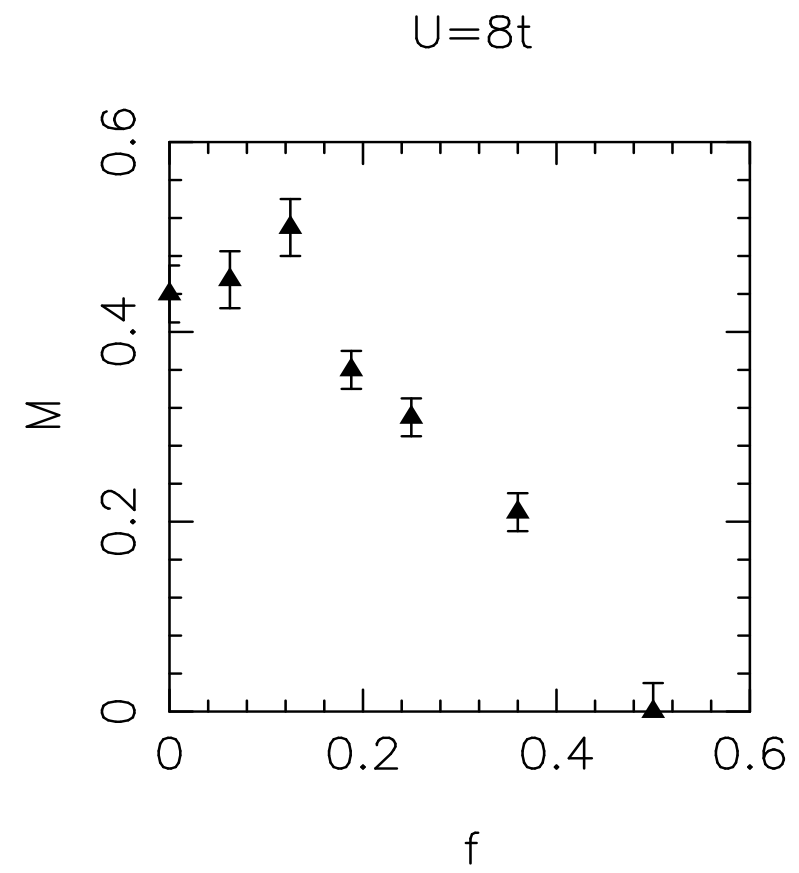

Figure 2: Ground-state staggered magnetization $M$ as a function of fraction $f$ of $U=0$ sites, as extrapolated from Fig. 1 .

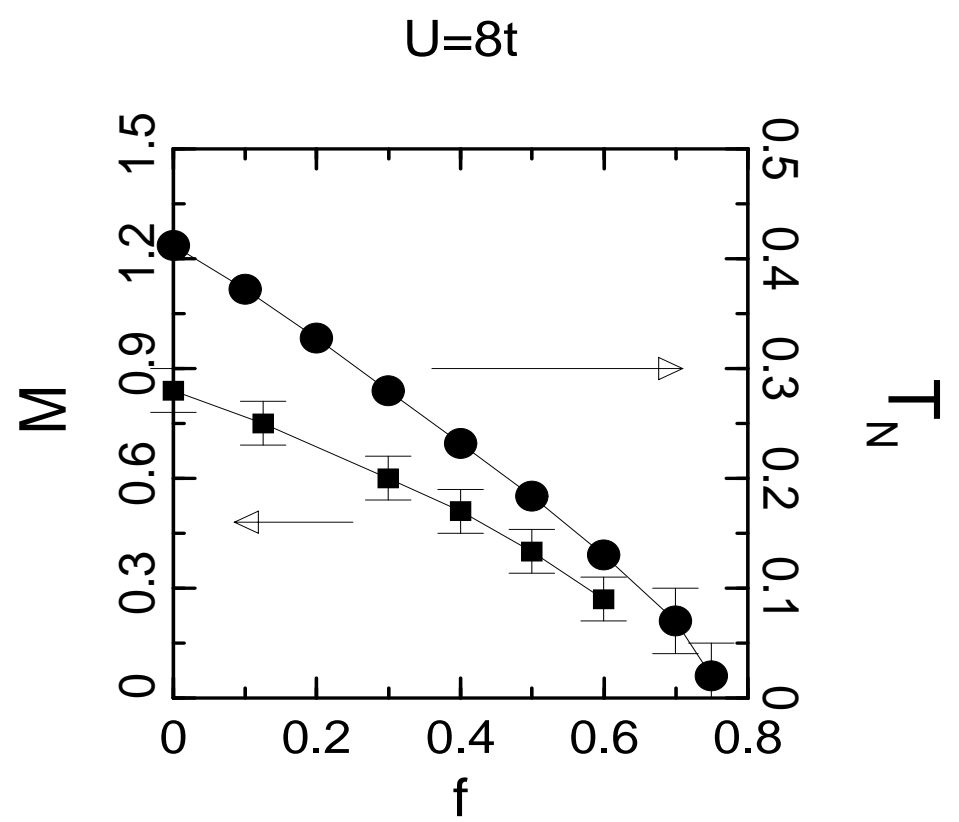

Figure 3: Staggered magnetization $M$ and Néel temperature $T_{\mathrm{N}}$ as a function of fraction $f$ of $U=0$ sites, computed using Dynamical Mean Field Theory (DMFT). $T_{\mathrm{N}}$ values are obtained by extrapolating $\Delta \tau$ to zero (see section 2.2) those for $M$ are not, since extrapolation is cumbersome. Also, small values of $M$ are hard to compute. 
To come to an explanation for this disorder-stabilized magnetic order we argue that the situation is different in one important aspect from the case of electronor hole-doping of the antiferromagnet at half-filling in the pure Hubbard model, which destroys AF order rapidly. In the pure case, the extra electrons (or: holes) are mobile and therefore particularly effective in destroying long-range order. With $U=0$ sites, however, extra particles will be localized on these sites, where they don't have to pay the on-site repulsion energy. So whereas magnetic moments are destroyed locally (on the $U=0$ sites), the long-range order originating from the $U \neq 0$ sites will be able to persist much longer. That $U=0$ sites are not so detrimental to AF order can also be seen from the fact that the usual perturbative argument to obtain an AF (superexchange) Heisenberg coupling in the pure model still goes through with an intermediate $U=0$ site. Finally, a qualitative way to understand the enhanced $M$ at $n=1$ comes from spin wave theory: normally the quantum fluctuations of spin waves reduce the magnetization. The effect of disorder can be thought of as to introduce a lifetime, which can be seen to reduce the effect of the spin waves on $M$, thereby enhancing it. Analogously, introducing $U=0$ sites into the negative- $U$ Hubbard model we expect to lead to enhanced charge- density wave order and superconductivity. The spin-wave argument may also explain the absence of the enhancement of $M$ in DMFT in Fig. 3: in this approach the coherentpotential approximation (CPA) is used to treat disorder (see section 2.2) and the CPA is known not to describe localization (in this case of spin waves), because it does not introduce spatial fluctuations 13 .

\subsection{Charge order}

A second type of order that is of interest is charge order, which generally is signalled by a range of constant density (or: zero compressibility $\kappa=\partial n / \partial \mu$ ) when the chemical potential is varied. For instance, in the Quantum Hall effects the occurrence of incompressible states is the central theme. In the pure Hubbard model a charge gap (proportional to $U$ ) occurs around $n=1$ : the system resists against having doubly occupied sites. To study the effect of disorder by having $U=0$ sites, we calculate the (disorder-averaged) density $n$ when varying $\mu$ in (2) at $U=8 t$ for a few values of impurity concentration $f$. To get a more detailed picture, we discriminate between $U=0$ and $U=8 t$ sites. The results are displayed in Figs. 4 to 7 .

Before embarking on a discussion of the results, we first discuss the specific averaging procedure that has been used to obtain the result for $n(\mu)$ in Figs. 4 and 5 (using Determinant QMC on $6 \times 6$ lattices). Because of the sign problem it is not possible to obtain reliable information at low enough temperatures on large lattices in the pure model for densities $1<n<1.3$ ([18], see also section 2.1). Introducing disorder turns out to help us in two ways: (i) in cases where the sign problem is less severe, e.g. for $n=1.4$, the computed average sign is closer to 1 in the presence of disorder, which means that the problem is more tractable, and (ii) the disorder average that one has to perform leads to a natural way of taming the sign problem. Concerning (ii): since for each realization of (quenched) disorder a relatively small

part of phase space (but hopefully large enough to be representative) is sampled, it can happen that for some realizations one is hit by the sign problem, but for others 


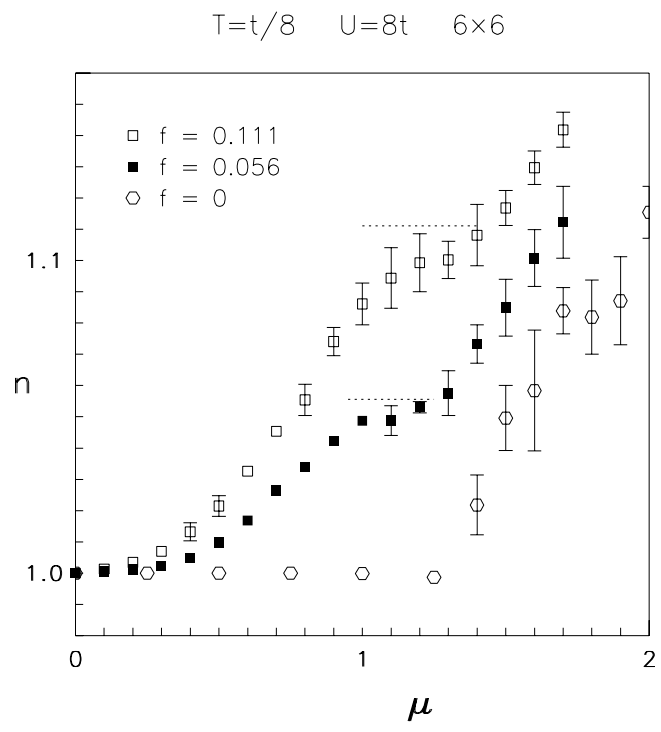

Figure 4: Average total density $n$ as a function of $\mu$ for different impurity concentrations $f=0,0.056,0.111$. Dotted lines indicate the corresponding values $1+f$. Calculated using Determinant QMC on $6 \times 6$ lattices with $U=8 t$ and $T=t / 8$. Error bars are within the symbol size when not shown.

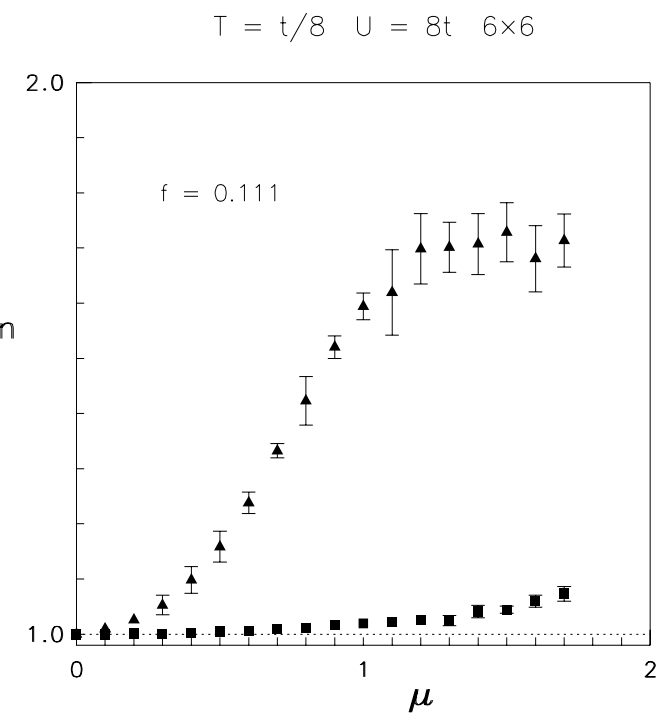

Figure 5: Average density $n$ on $U=0$ sites (triangles) and $U=8 t$ sites (squares) separately for impurity concentration $f=0.111$. The saturation value for $n(U=0)$ is about 1.71. Details as with Fig. 4. 


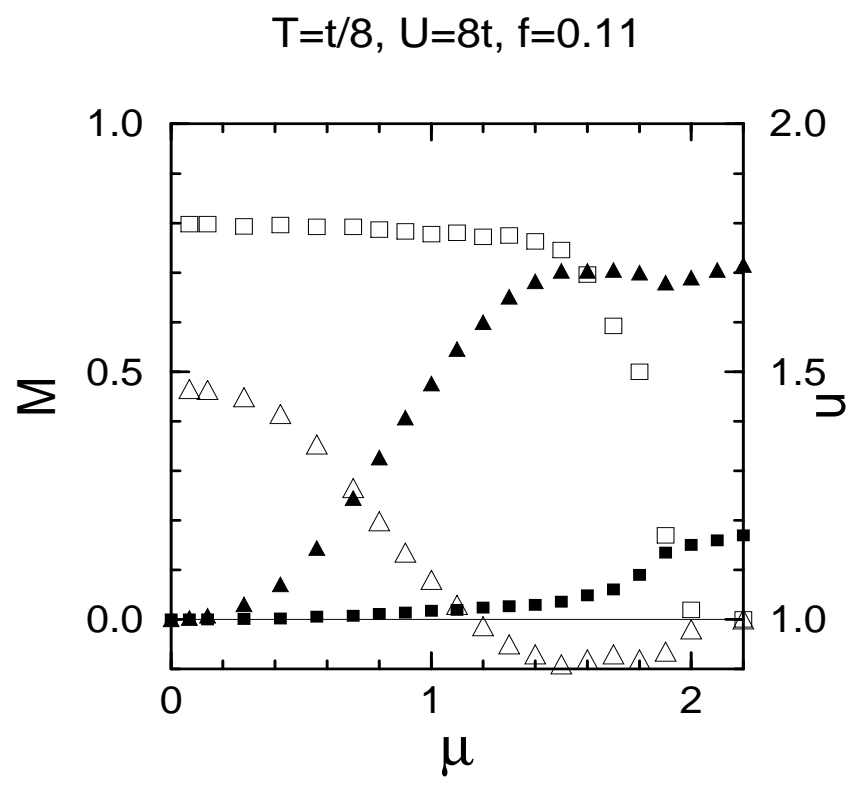

Figure 6: Average density $n$ (filled symbols) and sublattice magnetization $M$ (open symbols) on $U=0$ sites (triangles) and $U=8 t$ sites (squares) separately. The saturation value for $n(U=0)$ is about 1.70. Calculated using DMFT for impurity concentration $f=0.11, U=8 t$ and $T=t / 8$. Fig. 5 shows the corresponding results for $n$ for $d=2$.

$$
T=t / 8 \quad U=8 t
$$

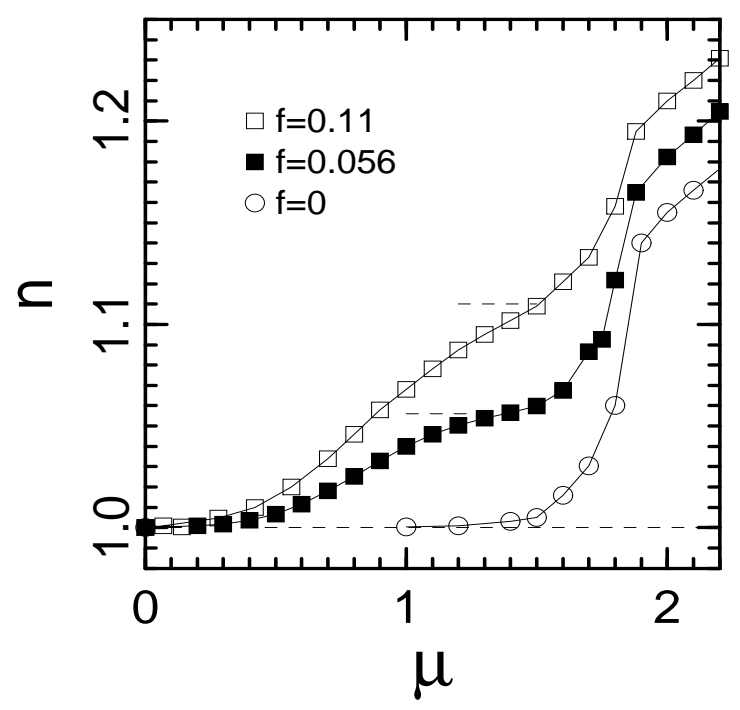

Figure 7: Average total density $n$ as function of $\mu$ for different impurity concentrations $f=0,0.056,0.11$, as calculated using DMFT. Dashed lines indicate the occurring values $1+f$. Details as with Fig. 6. Fig. 4 shows the corresponding results for $d=2$. 
one is not. This can be seen from the average sign which becomes very small, or from calculated quantities which occasionally deviate a lot from the majority of results (or even become unphysical, e.g. negative densities). To include such outliers in an average clearly makes no sense. A more reliable and robust way of determining the disorder average, i.e. one which is not very sensitive to the type of distribution which governs the outcomes, is the so-called midmean, or: $25 \%$ trimmed mean (an average over the middle half of the ordered data set [27]). We have used this mean for the $d=2$ QMC calculations; using another robust mean, the broadened median [27], gives very similar results. We like to stress that this approach is not limited to the disordered case; in fact, we have applied it to the pure $(f=0)$ model off half-filling as well (Fig. 4) by having different starting configurations take the role of different disorder realizations.

In Fig. 4, it is possible to discern for non-zero $f$ two flat regions in $n(\mu)$, i.e. two hardly compressible states. The first still occurs for $n=1$, but has a much reduced charge gap, whereas a second gap develops at a density somewhat below $n=1+f$. One would expect a plateau exactly at $n=1+f$ if the $U=0$ sites would fill independently of the $U \neq 0$ sites (because above $1+f$ the $U \neq 0$ sites would have to start to be doubly occupied). Since the density at $U=0$ sites does not saturate at 2 , but at a lower value $(n=1.76$ and 1.71 for $f=0.056$ and 0.111 , respectively; see Fig. 5), clearly the two sets of sites are coupled. On the other hand, one notes from Fig. 4 that the second plateau for non-zero $f$ terminates almost exactly at the $\mu_{\mathrm{c}}$ for $f=0$. Therefore, the chemical potential needed to force double occupation of the $U \neq 0$ sites is unaffected by the presence of $U=0$ impurities. The fact that $n$ at the $U \neq 0$ sites remains pinned at 1 (Fig. 5) up to about the same value of $\mu$ as for $f=0$ confirms this. As discussed above, indeed the error bars for non-zero $f$ are smaller than for the pure case, indicating a less-severe sign problem.

The remnant gap at $n=1$ is in fact the more remarkable one, since the gap at $n=1+f$ is a clear descendant of the one at $n=1$ in the pure model (see above). This can also be appreciated from considering our model first with $t=0$; for that case $n(\mu)$ will have two plateaus, at $n=1+f$ and $n=1-f$ (not mentioned above, but present because of particle-hole symmetry). Turning on the hopping not only leads to a rounding of the sharp steps, but also to the appearance of a new plateau at $n=1$. We argue the gap at $n=1$ to be due to induced AF order on the $U=0$ sites. To support this we have computed within DMFT for $f=0.11$ besides the densities also the sublattice magnetizations $M$ (Fig. 6). The decrease of $M$ is clearly coupled to the pinning of $n$ at 1 , both for $U=0$ and $U=8 t$ sites. For small $\mu$ there is AF order on both types of sites, whereas for $\mu>1.6$ the density on $U=0$ sites saturates, the density on $U=8 t$ sites starts to increase, and AF order disappears. Therefore, certainly for this rather large value of $U$, both charge gaps turn out to be intimately linked to AF magnetic order.

Somewhat surprisingly, $M$ on the $U=0$ sites becomes negative when $n$ on these sites saturates. The reason is that electrons at $U=0$ sites are more strongly localized when their spin is parallel to the neighbors than when it is antiparallel (because of Pauli's principle). Therefore, the net moment on $U=0$ sites is parallel to its neighbors, i.e. opposite to the total staggered magnetization.

For completeness we also show the total density as function of $\mu$ computed using 


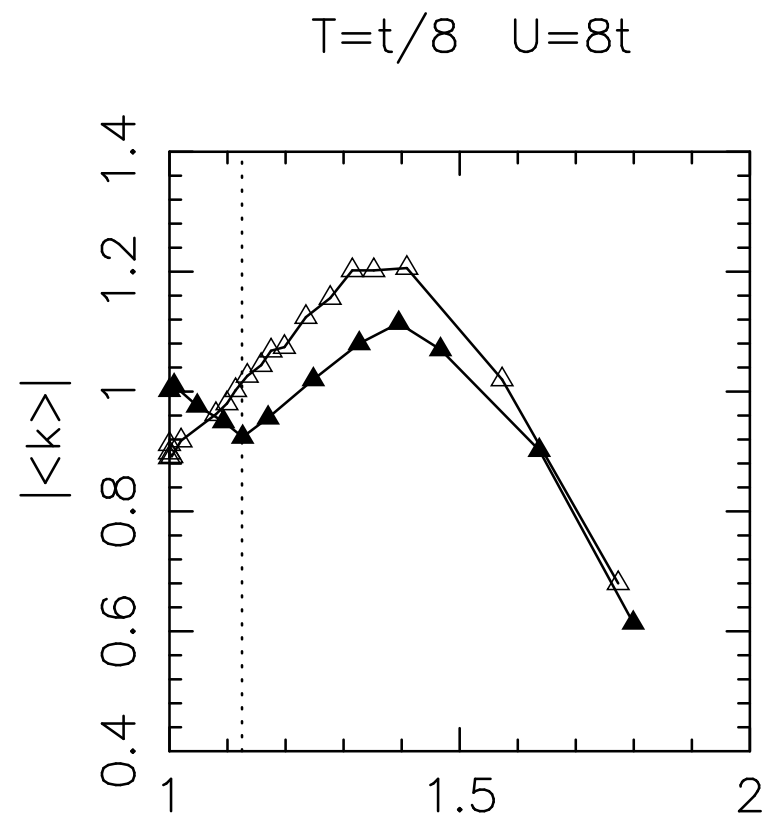

$\mathrm{n}$

Figure 8: Kinetic energy $|\langle K\rangle|$ as function of density $n$ for impurity concentrations $f=0$ (open triangles) and $f=0.11$ (filled triangles). Calculated using Determinant QMC on $4 \times 4$ lattices with $U=8 t$ and $T=t / 8$.

DMFT in the thermodynamic limit (Fig. 7). The overall picture of the $d=2$ QMC calculations on $6 \times 6$ lattices (Fig. 4) is confirmed, although the plateaus close to $1+f$ are less well developed, certainly for $f=0.11$. Another difference is that things happen at a slightly higher value of $\mu$, because fewer fluctuations are accounted for in DMFT.

Finally, we strengthen our case for an incompressible state close to $n=1+f$ by showing in Fig. 8 the kinetic energy (as computed on a $4 \times 4$ lattice) as function of $n$. Clearly there is a dip in the electron mobility at $n=1+f$. A similar argument, using cusps in the kinetic energy as evidence for incompressible states, was found to work for the boson Hubbard model [28]. We further note that additional support can be found in calculations of the density of states within DMFT that we present elsewhere [26].

In summary, using Determinant QMC calculations on finite lattices for $d=2$ combined with the infinite-dimension approach in the thermodynamic limit, we have studied the effect on ordered states in the Hubbard model of introducing disorder of the third kind, namely in the on-site interaction. We show that turning off the on-site interaction on a fraction of the sites of the lattice may enhance and stabilize AF order. It also leads to the occurrence of two incompressible states instead of one in the case without disorder; closer scrutiny of density and sublattice magnetization on the two types of sites teaches that both states are associated with AF order. 


\section{Acknowledgments}

This paper is dedicated to Hans van Leeuwen on the occasion of his 65th birthday. The first author thanks Hans for introducing him to the wonderful world of the Hubbard model and for encouragement, guidance, and collaboration during several years of studies of this model. He furthermore is grateful to the UC Davis Physics Department for hospitality during the period in which the presented work originated. The research described here is supported in part by NSF-DMR-95-28535.

\section{References}

[1] P.A. Lee and T.V. Ramakrishnan, Rev. Mod. Phys. 57, 287 (1985), D. Belitz, T.R. Kirkpatrick, Rev. Mod. Phys. 66, 261 (1994).

[2] A.N. Berker, Physica A 194, 72 (1993).

[3] M.C. Gutzwiller, Phys. Rev. Lett. 10, 159 (1963), J. Hubbard, Proc. Roy. Soc. (London) A276, 238 (1963), J. Kanamori, Progr. Theor. Phys. (Kyoto) 30, 275 (1963), P.W. Anderson, Phys. Rev. 115, 2 (1959).

[4] F. Gebhard, The Mott Metal-Insulator Transition, Springer Tracts in Modern Physics, vol. 137 (Springer, Heidelberg, 1997).

[5] M.P.A. Fisher, P.B. Weichman, G. Grinstein, and D.S. Fisher, Phys. Rev. B 40, 546 (1989).

[6] M. Ulmke, R.T. Scalettar, Phys. Rev. B 55, 4149 (1997).

[7] P.J.H. Denteneer, M. Blaauboer, J. Phys.: Condens. Matter 7, 151 (1995).

[8] G.T. Zimanyi, E. Abrahams, Phys. Rev. Lett. 64, 2719 (1990).

[9] E. Dagotto, Rev. Mod. Phys. 66, 763 (1994).

[10] R. Blankenbecler, D.J. Scalapino, R.L. Sugar, Phys. Rev. D 24, 2278 (1981), S.R. White, R.L. Sugar, R.T. Scalettar, Phys. Rev. B 38, 11665 (1988).

[11] W. Metzner and D. Vollhardt, Phys. Rev. Lett. 62, 324 (1989), A. Georges, G. Kotliar, W. Krauth, and M. Rozenberg, Rev. Mod. Phys. 68, 13 (1996).

[12] V. Dobrosavljević and G. Kotliar, Phys. Rev. Lett. 71, 3218 (1993).

[13] M. Ulmke, V. Janiš, and D. Vollhardt, Phys. Rev. B 51, 10411 (1995).

[14] H.F. Trotter, Proc. Amer. Math. Soc. 10, 545 (1959), M. Suzuki, Prog. Theor. Phys. 56, 1454 (1976), R.M. Fye, Phys. Rev. B 33, 6271 (1986), R.M. Fye and R.T. Scalettar, Phys. Rev. B 36, 3833 (1987).

[15] J.E. Hirsch, Phys. Rev. B 31, 4403 (1985).

[16] N.E. Bickers, D.J. Scalapino, and S.R. White, Phys. Rev. Lett. 62, 961 (1989). 
[17] G. Sugiyama and S.E. Koonin, Ann. Phys. 168, 1 (1986), R.M. Fye and J.E. Hirsch, Phys. Rev. B 38, 433 (1988), S. Sorella, S. Baroni, R. Car, and M. Parrinello, Europhys. Lett. 8, 663 (1989).

[18] S.R. White, D.J. Scalapino, R.L. Sugar, E.Y. Loh, Jr., J.E. Gubernatis, and R.T. Scalettar, Phys. Rev. B 40, 506 (1989).

[19] V. Janiš and D. Vollhardt, Phys. Rev. B 46, 15172 (1992).

[20] We choose a semi-elliptic DOS, $N^{0}(E)=\left[\left(2 t^{*}\right)^{2}-E^{2}\right]^{1 / 2} /\left(2 \pi t^{* 2}\right)$. For comparing with the square lattice $(z=4)$, we use $t^{*}=2 t$ and hence the DMFT calculations use the same bandwidth $(W=8 t)$ and second moment $\left(\left\langle E^{2}\right\rangle=4 t^{2}\right)$ of $N^{0}(E)$ as in $d=2$. DMFT results are fairly insensitive to the choice of DOS.

[21] V. Janiš and D. Vollhardt, Int. J. Mod. Phys. B 6, 731 (1992).

[22] A. Georges and G. Kotliar, Phys. Rev. B 45, 6479 (1992), M. Jarrell, Phys. Rev. Lett. 69, 168 (1992).

[23] J.E. Hirsch and R.M. Fye, Phys. Rev. Lett. 56, 2521 (1986).

[24] D.A. Huse, Phys. Rev. B 37, 2380 (1988).

[25] G.B. Martins, M. Laukamp, J. Riera, and E. Dagotto, Phys. Rev. Lett. 78, 3563 (1997).

[26] M. Ulmke, P.J.H. Denteneer, R.T. Scalettar, and G.T. Zimanyi, in preparation.

[27] J.L. Rosenberger and M. Gasko, Ch. 10 in Understanding Robust and Exploratory Data Analysis, edited by D.C. Hoaglin, F. Mosteller, and J.W. Tukey (Wiley, New York, 1983).

[28] G.G. Batrouni, R.T. Scalettar, and G.T. Zimanyi, Phys. Rev. Lett. 65, 1765 (1990). 